\title{
Cálculo de trayectorias para vehículos autónomos marinos (USV) utilizando el método Fast Marching Square sometido a campo vectorial
}

\author{
Melchor Izquierdo Sánchez, 100283545@alumnos.uc3m.es \\ Santiago Garrido, sgarrido@ing.uc3m.es \\ Luis Moreno, moreno@ing.uc3m.es
}

\section{Resumen}

La planificación de trayectorias fue en el pasado y es actualmente un campo de estudio con amplias posibilidades y muy investigado. Para resolver los problemas planteados existen diferentes aproximaciones y algoritmos, entre los cuales el Fast Marching Method y el Fast Marching Square han ofrecido muy buenos resultados con respecto a los indicadores de rendimiento de estos métodos (seguridad y suavidad de trayectoria, fiabilidad, bajo carga computacional y robustez del algoritmo).

En este documento se explora la utilización del Fast Marching sometido a Campo Vectorial (FMVF) en aplicaciones para Vehículos Autónomos de Superficie (USVs), a partir del Fast Marching Square. Éste tiene en cuenta las acciones del entorno sobre el robot o vehículo, diferentes dependiendo de la aplicación, mejorando de esta manera el comportamiento en condiciones reales y el desempeño.

Palabras clave: Fast Marching, Fast Marching Square, Fast Marching subject to Vectorial Field, Unmanned Surface Vehicle.

\section{INTRODUCCIÓN}

El objetivo de este documento es el análisis de la utilización del método Fast Marching Square (FM2) con influencia de un campo vectorial externo en el cálculo de trayectorias de vehículos marinos autónomos (USVs, Unmanned Surface Vehicles) en entornos en los que las corrientes superficiales de agua puedan afectar a la trayectoria.

Un algoritmo de planificación de trayectorias ideal se caracterizará por características como: seguridad, tiempo de computación reducido, eficiencia, proporcionar trayectorias alisadas, etc.

El algoritmo debe ser válido para diferentes ámbitos y aplicaciones, haciendo sencilla su implantación en diferentes situaciones, sin necesidad de hacer modificaciones para adaptarlo. Es importante también que el tiempo de computación sea reducido para permitir una adaptación en tiempo real a posibles cambios en el entorno y evitar a tiempo posibles colisiones recalculando y modificando la trayectoria.

\section{MÉTODO}

En este texto, el método empleado es el Fast Marching Method (FM), del que se deriva el método Fast Marching Square $\left(\mathrm{FM}^{2}\right)$, y de éste el Fast Marching sometido a Campo Vectorial (FMVF). Dentro de ese campo vectorial se pretende modelar la influencia de agentes externos sobre la trayectoria óptima entre dos puntos.

Estos métodos emplean campos potenciales artificiales creados sobre un mapa del entorno para realizar el cálculo de la trayectoria óptima en este mapa en condiciones de seguridad y evitando colisiones con los obstáculos. La creación de los campos potenciales se lleva a cabo mediante el uso del Fast Marching, de manera que se consigue evitar mínimos locales, evitando así trampas en las que el vehículo quede atascado sin encontrar la solución al problema. El FM ${ }^{2}$ se basa en el tiempo de llegada de una onda en expansión desde el punto de partida del robot hasta cada punto del mapa, creando así un mapa en el que puede seguirse el camino en el que se emplea el menor tiempo en recorrido. Además, como el tiempo de computación es reducido, lo cual hace posible recalcular la trayectoria constantemente desde la posición actual del vehículo hasta la posición objetivo, de manera que si se presenta un obstáculo de manera repentina, el método es capaz de evitar la colisión y recalcular la trayectoria.

\subsection{Método Fast Marching}

Es un método desarrollado por J. Sethian y S. Osher [1], y es un caso especial de Método de Conjunto de Nivel (Level Set Methods).

Es un algoritmo numérico de resolución rápida, basado en la expansión de un frente de onda en un medio no homogéneo. Existen otros métodos de resolución similares basados en el mismo concepto, aunque son de más lenta resolución. Aparte del uso al que se le somete en este texto, es un 
método que puede ser empleado en aplicaciones diferentes a ésta, como en problemas de geometría, mecánica, visión por ordenador, etc.

\subsection{Introducción a Fast Marching y Fast Marching Square}

[2] En la naturaleza, una onda (como por ejemplo un rayo de luz) encuentra el camino de menor tiempo posible, debido al principio de Fermat. La propagación de la onda en el medio es proporcional a la velocidad de propagación en éste y al índice de refracción.

La propagación de esta onda está caracterizada por la ecuación de la Eikonal. Si sólamente existe un punto fuente de luz/onda, cada punto estará conectado con el este origen mediante un camino asociado con el parámetro tiempo. El conjunto de puntos del dominio con el tiempo como tercera coordenada en el caso de un problema bidimensional resulta en una superficie de Lyapunov. En esta curva de Lyapunov, las curvas de nivel son isócronas, correspodiendo a diferentes tiempos, y los caminos de Fermat son ortogonales a esas curvas de nivel. De esta manera, no es posible obtener mínimos locales.

El Fast Marching Method consiste en la propagación de una onda desde los obtáculos y las paredes del mapa. De esta manera se obtiene una matriz $\mathrm{W}$ que proporciona el índice de refracción, o potencial de lentitud, o matriz de dificultad o viscosidad. Este es el llamado primer potencial, que suele representarse en escala de grises correspondiendo el negro a velocidad nula y el blanco a velocidad máxima. En este primer potencial, pueden emplearse distintos niveles de saturación, de manera que puede variarse el nivel de seguridad, exactitud, ajuste a los obstáculos, etc. Desde el primer potencial, al llevarse a cabo la expansión de onda, se obtiene una superficie de Lyapunov, o segundo potencial. Entonces, para obtener el camino de mínimo tiempo, se aplica el camino de máxima pendiente entre las superficies isócronas de la superficie de Lyapunov.

\subsection{Ecuación de la Eikonal}

[3] El frente de onda puede ser caracterizado mediante el tiempo de llegada al punto en el que se realiza el cálculo, T.

$$
\theta=F T
$$

En la ecuación 1, la distancia es $\theta$, y $\mathrm{F}$ la velocidad del frente de onda.

De la ecuación 1, la derivada espacial es el gradi-

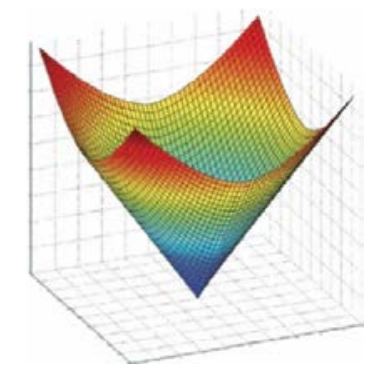

Figura 1: Superficie de Lyapunov. La dimensión relativa al tiempo es la vertical y las dos dimensiones horizontales corresponden a las espaciales

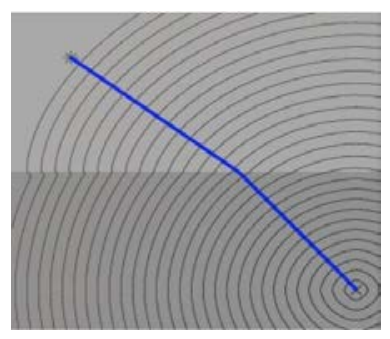

(a)

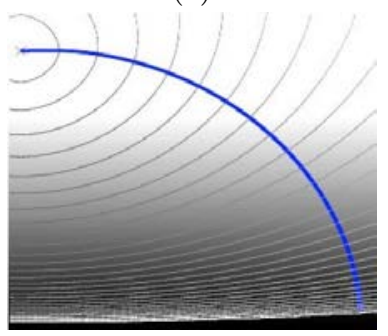

(b)

Figura 2: Trayectorias obtenidas mediante FM para dos medios con diferentes índices de refracción (2a) y para un medio contínuo cuyo índice de refracción va en aumento (2b) de arriba hacia abajo

ente de la ecuación 2:

$$
1=F \frac{d T}{d \theta}
$$

Por lo tanto, la magnitud de la función tiempo de llegada $\mathrm{T}$ en función del espacio $\theta$ es inversamente proporcional a la velocidad de propagación $\mathrm{F}$.

$$
\frac{1}{F}=|\nabla T|
$$

Para un problema multidimensional, el movimiento del frente es la solución de un problema de condiciones de contorno, debido a que las curvas de nivel isócronas son ortogonales al gradiente de expansión de la onda.

Si la velocidad de propagación $\mathrm{F}$ de la onda es solamente función de la posición $\theta$ en la anterior ecuación 3 , puede expresarse como la ecuación de la Eikonal:

$$
|\nabla T| F=1
$$




\subsection{Método}

El Fast Marching Method es un método numérico para resolver esta ecuación de la Eikonal (4) desarrollado por Sethian [4], inicialmente empleando mallas rectangulares. Es un algoritmo $\mathrm{O}(\mathrm{n})$, donde $n$ es el número total de puntos de la malla, en el que el método sube por la malla de manera similar al algoritmo de Dijkstra. Ésto significa que es un algoritmo de orden de complejidad lineal, por lo que la relación entre el número de puntos y el tiempo de computación es lineal, o de otra manera, si el mapa a computar tiene el doble de puntos que otro, el cálculo llevará el doble de tiempo. Ello contrasta con con los órdenes de complejidad de otros algoritmos como exponencial para $A^{*}$ (generalmente, sin empleo de método heurístico), asintótica para RRT, o entre parabólica y logarítmica para Roadmaps.

El algoritmo fue diseñado para problemas en los que la velocidad de propagación $\mathrm{F}$ del frente de onda no cambia de signo, esto significa que la onda sólo se mueve hacia adelante o atrás, sin existir reflexión o rebote. De esta manera, se consigue transformar la tarea en un problema estacionario, lo cual tiene dos implicaciones: el tiempo de computación se ve reducido de manera considerable, y el frente de onda solamente pasa una vez por cada punto del espacio/malla.

En cuanto a rapidez de respuesta, el método debe ser capaz de calcular la ruta de manera lo suficientemente rápida para evitar posibles imprevistos. Por esta razón, la información proveniente de los sensores ha de ser tratada de manera rápida y simple.

Además, el planificador debe ser capaz de proporcionar una trayectoria suave, para que ésta pueda ser ejecutada por el controlador de movimientos del vehículo, evitando así la necesidad de refinar la trayectoria posteriormente.

La fiabilidad de las trayectorias obtenidas afecta directamente a la distancia del robot o vehículo con respecto a los objetos o paredes y la velocidad de avance para cada punto de la malla. Esto último es una ventaja de método Fast Marching, el cual presenta también el perfil de velocidades para cada momento que hace posible realizar la trayectoria objetivo de manera segura.

Finalmente, otra de las propiedades del Fast Marching Method es que al estar basado en la propagación de un frente de onda, encuentra siempre la solución al problema si ésta existe.

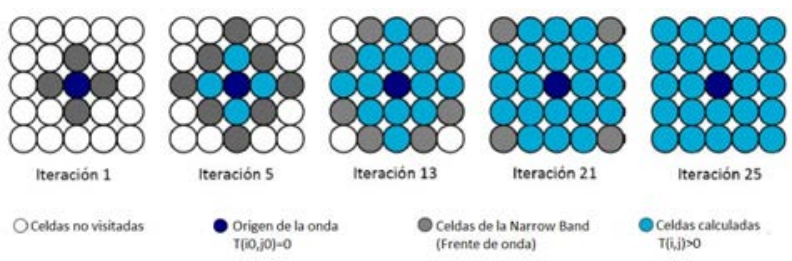

(a) Iteraciones en Fast Marching

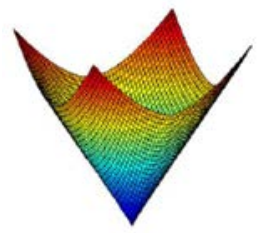

(b) Superficie de Lyapunov con un foco

Figura 3: Implementación de FM2

\subsection{Implementación de FM2}

[5] El método fue en origen desarrollado para mallas ortogonales. Se emplea en situaciones similares a la propagación frontal de una onda, con una función de velocidad dependiente de la posición, $\mathrm{F}=\mathrm{F}_{(\mathrm{i}, \mathrm{j})}$. La solución al problema se construye siempre avanzando solamente "hacia arriba", cumpliendo la llamada "condición de entropía". El método cumple además el Principio de Fermat, por el cual la luz, o una onda, viaja siempre entre dos puntos por el camino más rápido posible.

Si tomamos $\mathrm{T}_{(\mathrm{i}, \mathrm{j})}$ como la superficie solución en la que la curva corta el punto (i,j) del espacio, entonces se satisface la solución de la Eikonal (ec. 4). El valor de $\mathrm{T}$ siempre va en aumento, de acuerdo con lo anteriormente expresado.

En la malla existirán tres tipos de puntos: puntos que no han sido aún visitados por el frente de onda, puntos que están siendo calculados en el momento (en otras palabras, puntos que forman parte del frente de onda para ese instante, llamada Narrow Band) y puntos que ya han sido visitados y computados y que tienen asignado un valor de tiempo de llegada del frente y cuyo valor ya no podrá variar posteriormente. En cada iteración, el punto perteneciente al frente de onda con un valor de tiempo de llegada menor se marcará como ya visitado y se caracterizará con ese valor de tiempo, quedando éste fijo, y los puntos adyacentes a éste se marcarán como puntos pertenecientes al frente de onda. Este funcionamiento se repite, expandiéndose desde el punto de origen inicial hasta que todos los puntos de la malla estén caracterizados como puntos ya visitados y computados.

Una de las características del método es que puede aplicarse a mallas triangulares, a diferencia del 
método de Dijkstra, que restringe su camino a las aristas entre nodos. En el Fast Marching Method, el camino puede atravesar las caras triangulares de la malla, por lo que un vértice o nodo puede ser actualizado desde un triángulo. Para ello, necesita 2 vértices de apoyo. Así, cuando el frente de onda llega a un punto $\mathrm{x}_{1}$ a un tiempo $\mathrm{T}_{(\mathrm{x} 1)}$ y a otro punto $\mathrm{x}_{2}$ a $\mathrm{T}_{(\mathrm{x} 2)}$, el paso de activación puede estimar cuándo llegará el frente a $x_{3}$.

\subsection{Fast Marching Square sometido a campo vectorial externo, FMVF}

Método desarrollado por Vladimirsky [6] como Anisotropic Fast Marching, aunque ese mismo nombre había sido utilizado por Peyre [7] para otro método. Teniendo en cuenta que Valero había utilizado ya la nomenclatura Vectorial Fast Marching [8] para otro tipo de aplicaciones empleando Fast Marching, Garrido, Moreno y Alvarez [9] utilizaron Fast Marching sujeto a Campo Vectorial, para corresponder al sentido físico del método.

Éste construye una función de coste $f=1-n$. Como la velocidad del robot varía en el rango [0, $\mathrm{V}]$, siendo $\mathrm{V}$ la velocidad máxima de éste, y el índice de refracción es $n=c / F$, siendo c la velocidad de la luz en vacío y $\mathrm{F}$ la velocidad en el medio, el índice de refracción varía en un rango $[0,1]$ en el que 0 corresponde al vehículo parado y 1 a éste viajando a velocidad máxima. Así, la función de coste $f$ varía en el intervalo $[0,1]$, en el que 0 es el coste mínimo y 1 el máximo. La ruta óptima es la que hace que el tiempo entre el punto inicial $\left(\mathrm{x}_{\mathrm{s}}\right)$ y el final $\left(\mathrm{x}_{\mathrm{g}}\right)$ sea mínimo.

$$
T=V \min _{C(s) \subset D} \int_{x_{\mathrm{S}}}^{x_{\mathrm{g}}} f(\mathrm{C}(\mathrm{s})) d s
$$

En la ec(5), C(s) es el camino en el dominio D.

Hay que destacar que Vladimirsky [6] o Petres $[10,11]$ abandonaron esta técnica, lo que Garrido, Moreno y Álvarez [9] creen que se debe a que normalizaban la magnitud del campo vectorial sin tener en cuenta la magnitud de la función de coste, anulando así el efecto de campos vectoriales superiores a la unidad sobre el camino calculado, por lo que ellos normalizan la función coste:

$$
\widetilde{f}=f_{\text {dif }}+f_{\text {vect }}
$$

En la ecuación $6, \mathrm{f}_{\text {dif }}$ correponde al factor de dificultad, siendo $f_{\text {dif }}=1-W, \mathrm{y} \mathrm{fect}_{\text {vect }}$ corresponde a la componente vectorial.

Cabe destacar que $\tilde{f}$ es siempre positiva, debido a que en Fast Marching la onda siempre avanza en

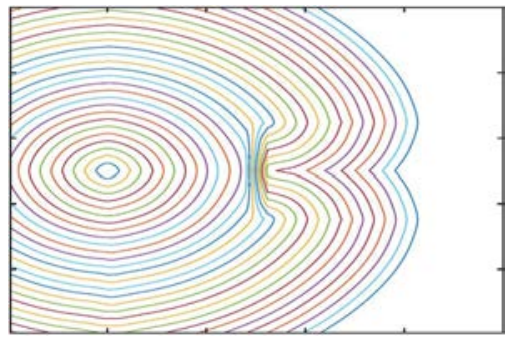

(a)

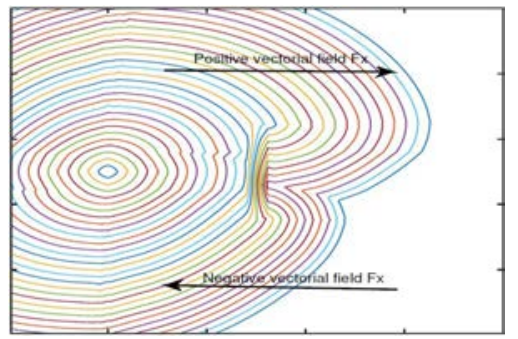

(b)

Figura 4: Expansión de una onda en FM con un obstáculo rectangular

un sentido sin retroceder.

En la figura 4, puede observarse la diferencia entre una onda en expansión al encontrarse con un obstáculo rectangular en dos instancias diferentes. Entre la primera imagen 4a y la segunda, 4b, la diferencia es la presencia del campo vectorial, el cual modifica la expansión del frente de onda.

En la matriz de dificultad, W, pueden incluirse diferentes atributos dependiendo de las características del terreno, la orografía, y otras preferencias mediante la inclusión de unos factores, los cuales pueden modificarse en su valor para primar ciertos aspectos de los anteriores sobre otros en el cálculo de la matriz de dificultad y, por lo tanto en la trayectoria.

$$
W=a_{1} A+a_{2} B+\ldots
$$

Donde:

$$
\sum a_{\mathrm{i}}=1
$$

En esta Matriz W pueden primarse diferentes aspectos del camino sobre los que el algoritmo hallará el camino de mínimo coste, como longitud, cercanía a obstáculos, energía, gradiente de pendiente, etc. Ello depende de la clase de vehículo a la que esté aplicado (terrestres, marinos, submarinos, aéreos, etc). 


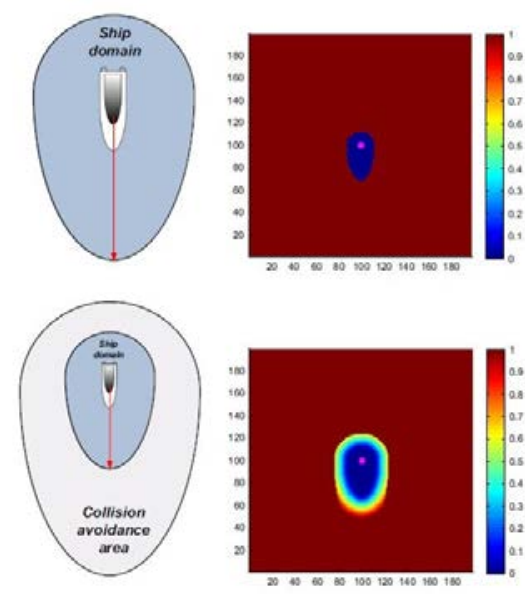

(a) Dominio y zona de evasión

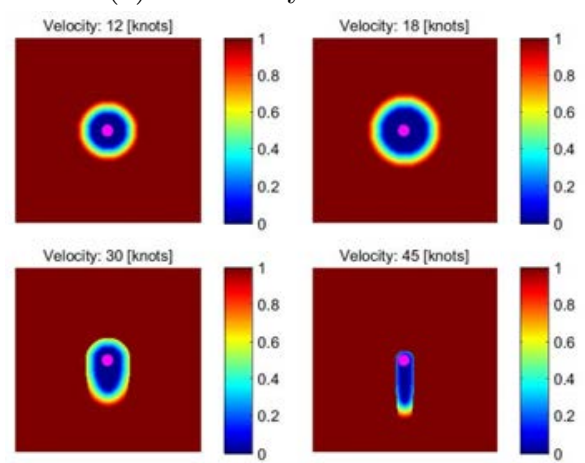

(b) Forma de zona para varias velocidades

Figura 5: Dominio de la embarcación y zona de evasión de colisiones en diferentes casos

\subsection{Aplicación a embarcaciones}

La mayoría de trabajos previos en este campo $[13,14,15]$ se restringían a entornos simples, pero otros trabajos $[12,16]$ lo orientaron a entornos oceánicos más cercanos a la realidad, con obstáculos estáticos y dinámicos. Liu y Bucknall [12] modelan una embarcación de manera dinámica, empleando como punto de partida la forma propuesta por Tam y Bucknall [17], con la diferencia de que en su caso, la forma del dominio de la embarcación y la zona de evasión de colisiones (figura 5a) varía en función del perfil de velocidad del vehículo, de manera que esta forma se estiliza a la vez que se aumenta el módulo de la velocidad (figura 5b). Éste comportamiento pude observarse en la figura 5. Éste método es más ajustado a la realidad que otros, como el implementado por Yang, Tseng y Tseng [18], simplemente limita el área de seguridad a un rectángulo imaginario con sus magnitudes relativas a las dimensiones de la embarcación, más simple y menos ajustado a la realidad.

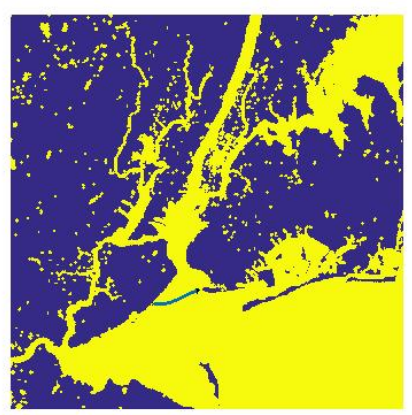

Figura 6: Trayectoria del USV en la Upper Bay sin presencia de mareas

\section{SIMULACIONES}

Este apartado se centra en aplicaciones para USVs, en dos tipos de entorno diferentes: estuarios de ríos donde la acción de las mareas es importante, y entornos de aguas abiertas donde las corrientes superficiales de agua afectan a la trayectoria del vehículo.

Para generar el mapa de fuerzas vectoriales que caracteriza la corriente, se realiza una expansión desde los límites del mapa, los cuales corresponden al mar, que se expande como una onda hacia el interior del río, resultando en un mapa en el que el módulo de la corriente es mayor en el mar y menor cuanto más se adentra en el río y cuanto más cercano está de la orilla.

\section{$3.1 \quad$ Nueva York}

Los ensayos se realizaron utilizando un mapa del estuario del río Hudson, en la ciudad de Nueva York.

Empleando esto, pueden realizarse cálculos de trayectorias para corrientes con distintas fuerzas. En la figuras 6 y siguientes se puede observar el efecto de la fuerza vectorial sobre la trayectoria calculada para unos mismos puntos de inicio y fin de camino. En las figuras 6, 7 y 8, se muestra la solución de aplicar el algoritmo a distintos puntos de inicio y final de trayectorias, en una zona angosta de la bahía.Tal y como está representado en la figura 8, las trayectorias varían cuando la constante que controla la fuerza de la corriente se ve aumentada. Las diferentes fuerzas de corriente aplicadas se obtienen fácilmente con la única variación de un parámetro, el cual multiplica la marea por este parámetro, que puede ser positivo o negativo, dependiendo de si esta marea está subiendo hacia el río o bajando desde él. En esta imagen, el valor de las corrientes con la nueva constante es comparado con el de la trayectoria para 


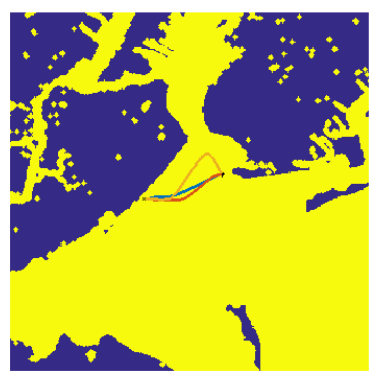

Figura 7: Comparación de las trayectorias para diferentes mareas crecientes con el caso base (figura 6)

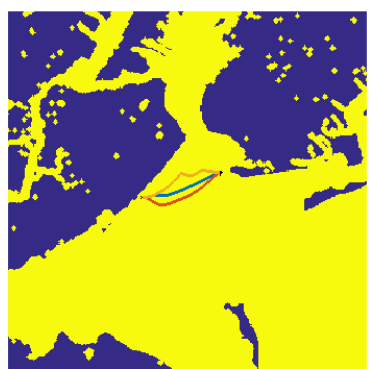

Figura 8: Comparación de las trayectorias para diferentes mareas decrecientes con el caso base (figura 6)

campo nulo, y en ella se muestran los resultados obtenidos al aplicar distintas constantes de fuerza al sistema.

Tanto en la figura figura 7 como en la figura 8 , se observa cómo el efecto de empuje del agua en la superficie desplaza al vehículo de manera que la trayectoria óptima se ve modificada con respecto a la original.

\subsection{Mar abierto - Trayectorias largas}

Este mismo método es aplicable a situaciones fuera de la acción de las mareas en desembocaduras de ríos, como el cálculo de trayectorias para vehículos en aguas abiertas. Para ello, es necesario emplear datos de las corrientes marinas u oceánicas, las cuales tienen diferentes magnitudes y direcciones dependiendo del punto. Esta información se ha tomado de un conjunto de datos descargados de la página web http://www.aoml.noaa.gov/phod/ dac/dac_meanvel.php [19, 20], del Laboratorio Meteorológico y Oceanográfico Atlántico, de la Administración Nacional Oceánica y Atmosférica norteamericana. Las mediciones de los datos de este conjunto están proporcionadas por boyas

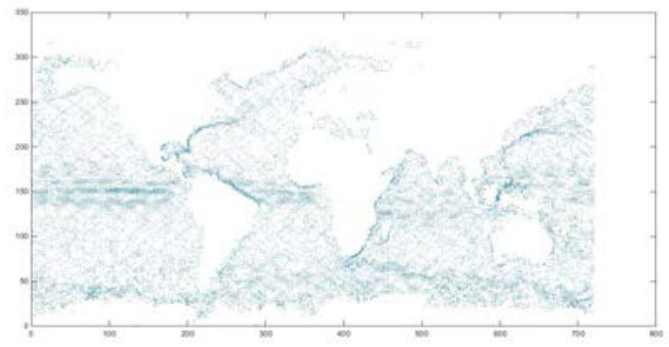

(a) Mapa de velocidades superficiales

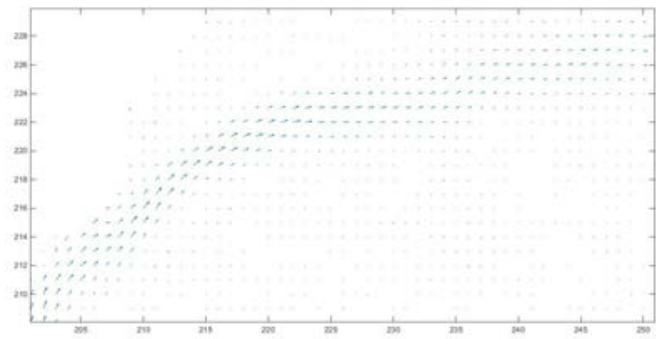

(b) Detalle de 9a

Figura 9: Mapa de velocidades superficiales del agua a partir de los datos obtenidos de los "drifters" y detalle del mismo

flotantes SVP (Surface Velocity Program), o SVP drifting buoys con seguimiento por satélite, con las que se realizan mediciones de las corrientes superficiales.

Puede observarse más fácilmente en una representación de los datos como vectores en cada punto. De esta manera, se obtiene un mapa que caracteriza la fuerza vectorial externa aplicada al método FMM para obtener la trayectoria más rápida (de menor coste de tiempo) entre los puntos de inicio y fin del trayecto. Estos mapas de vectores pueden observarse en la figura 9 .

Para la primera prueba se toma como puntos de inicio y final de recorrido dos puntos en Sudamérica y África, separados por el océano Atlántico. Éstos se corresponden aproximadamente a la costa entre Costa de Marfil y Ghana y la costa entre Surinam y la Guayana Francesa. En la imagen 10a se muestra el resultado obtenido al aplicar el método FMM sin incluir la interacción con una fuerza externa entre los dos puntos anteriormente mencionados.

A la vez, en ilustración 10b puede verse la respuesta al incluir la acción de la velocidad superficial del agua. Éstas modifican el camino de mínimo peso en cuanto a tiempo de forma que la trayectoria se desvía hacia el Sur. Esto concuerda con las corrientes en esta parte del Atlántico, las que por lo general son de dirección Oeste (en algunas zonas en dirección Oeste-Suroeste), siendo en 


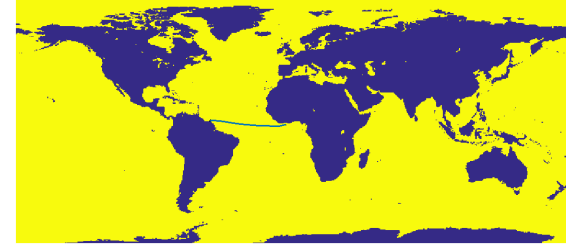

(a) Trayectoria obtenida mediante FM2, sin la acción de la velocidad superficial del agua

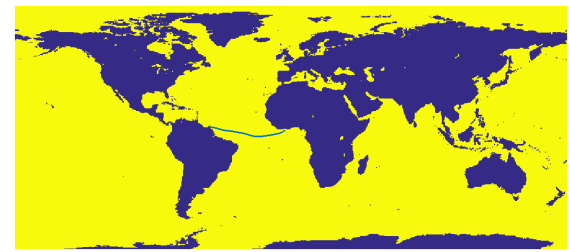

(b) 1m1Trayectoria obtenida mediante FVF2, con la influencia de corrientes superficiales

Figura 10: Simulación de travesía por el Atlántico entre África y América

el inicio (África) de dirección Este y en el final (Sudamérica) de dirección Oeste-Noroeste, siguiendo la línea de la costa hacia arriba.

Se realizó un segundo grupo de pruebas conectando dos puntos en el mar Caribe. El camino recorrido es el que une Nueva Orleans con Cartagena, Colombia. Comparando ambas figuras (11a con 11a) se percibe la acción de la corriente existente entre la isla de Cuba y Honduras, Belice y la península de Yucatán, en dirección Oeste y desde el mar Caribe hacia el golfo de México. Esta influencia, desvía la trayectoria hacia el Oeste tras haber rebasado la costa de Cuba, en el Canal de Yucatán.

\section{CONCLUSIONES}

Tras los experimentos realizados, los resultados fueron satisfactorios en la medida en que el algoritmo encuentra la solución al problema de hallar el camino más eficiente entre los puntos iniciales y finales de una trayectoria deseada. Ésto, combinado con la capacidad de recalcular muy regular o habitualmente la trayectoria, hace que el método se comporte de manera muy apropiado, haciéndolo capaz de reaccionar a cambios en el entorno, tanto obtáculos u otros vehículos que interfieran en el camino como en las condiciones de la superficie del agua.

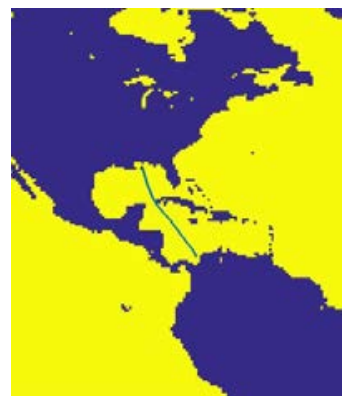

(a) Trayectoria base obtenida mediante FM2

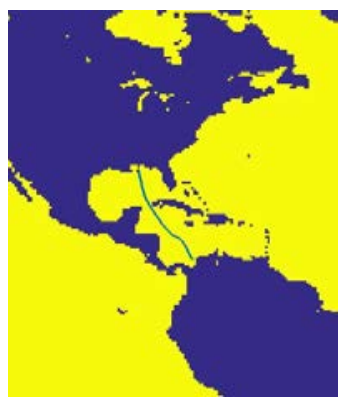

(b) Trayectoria afectada por las corrientes superficiales

Figura 11: Resultado de las pruebas realizadas entre Nueva Orleans y Cartagena

\section{Referencias}

[1] OSHER, Stanley; SETHIAN, James A., Noviembre 1988. Fronts Propagating with Curvature-dependent Speed: Algorithms Based on Hamilton-Jacobi Formulations. Journal of Computational Physics. Elsevier, vol. 79, no. 1, pp. 12-49. ISSN 0021-9991.

[2] GARRIDO, Santiago; MORENO, Luis E.; GÓMEZ, Javier V, 2015. Motion planning using Fast Marching Square method. En CARBONE, Giuseppe; GOMEZ-BRAVO, Fernando. Motion and Operation Planning of Robotics System: Background and Practical Approaches. Mechanics and Machine Science, vol. 29. Springer, pp. 223-248. ISBN 978-3319-14704-8.

[3] SETHIAN, James A., 1996. Level Set Methods and Fast Marching Methods. 2 Edición. Cambridge University Press, ISBN 9780521645577.

[4] SETHIAN, James A., Febrero 1996. Level Set Method for Monotonically Advancing Fronts. Proceedings of the National Academy of Sciences of the United States of America (Proc Natl Acad Sci), vol. 93, no. 4, pp. 1591-1595. ISSN 0027-8424.

[5] SETHIAN, James A., 1999. Fast Marching Methods and Level Set Methods for Propagat- 
ing Interfaces. Cambridge University Press, ISBN 978-0521645577.

[6] VLADIMIRSKY, Alexander; SETHIAN, James A., 2003. Ordered upwind methods for static Hamilton- Jacobi equation: Theory and algorithms. SIAM Journal of $\mathrm{Nu}$ meric Analysis. Society of Industrial and Applied Mathematics, vol. 41, no. 1, pp. 325363. ISSN 0036-1429.

[7] PEYRÉ, Gabriel, 2010. The Numerical Tours of Signal Processing - Advanced Computational Signal and Image Processing. IEEE Computing in Science and Engineering, vol. 13, no. 4, pp. 94-97. ISSN 1521-9615.

[8] VALERO GÓMEZ, Alberto; GARRIDO, Santiago; MORENO, Luis, 2013. The Path to Efficiency: Fast Marching Method for Safer, More Efficient Mobile Robot Trajectories. IEEE Robotics and Automation Magazine. IEEE, vol. 20, no. 4, pp. 111-120. ISSN 1070-9932.

[9] GARRIDO, Santiago; MORENO, Luis E.; ÁLVAREZ, David, 14-15 octubre 2015. Applying The Fast Marching Method To Path Planning On Mars. En IET, IMechE, SSI and SMESTECH Space Robotics Symposium - Present And Future Robotics In Space Applications. Glasgow. Disponible en: https: //www.researchgate.net/publication/ 282857265_APPLYING_THE_FAST_MARCHING_ METHOD_TO_PATH_PLANNING_ON_MARS.

[10] PÊTRÈS, Clément; PAILHAS, Yan; PETILLOT, Yvan; LANE, Dave, Junio 2005. Underwater Path Planning Using Fast Marching Algorithms. Oceans European Conference. Brest, Francia: IEEE, vol. 2, no. 1, pp. 814-819. ISBN 0-7803-9103-9.

[11] PTRS, Clément; PAILHAS, Yan; PATRON, Pedro; PETILLOT, Yvan; EVANS, Jonathan; LANE, David, Abril 2007. Path Planning for Autonomous Underwater Vehicles. Path Planning for Autonomous Underwater Vehicles. IEEE, vol. 23, no. 2, pp. 331341. ISSN 1552-3098.

[12] LIU, Yuanchang; BUCKNALL, Richard, Marzo 2015. Path Planning Algorithm for Unmanned Surface Vehicle Formations in a Practical Maritime Environment. Ocean Engineering. Pergamon, vol. 97, no.1, pp. 126144. ISSN 0029-8018.

[13] TAM, CheeKuang; BUCKNALL, Richard, Enero 20013. Cooperative Path Planning Algorithm for Marine Surface Vessels. Ocean
Engineering. Pergamon, vol. 57, no.1, pp. 2533. ISSN 0029-8018.

[14] NAEEM, Wasif; IRWIN, George W.; YANG, Aolei, Septiembre 2012. COLREGs-based Collision Avoidance Strategies for Unmanned Surface Vehicles. Mechatronics. Elsevier, vol. 22 , no. 6, pp. 669-678. ISSN 0957-4158. Mechatronics, 22, 669678, 2012.

[15] THAKUR, Atul; SVEC, Petr; GUPTA, Satyandra K., Diciembre 2012.GPU Based Generation of State Transition Models Using Simulations for Unmanned Surface Vehicle Trajectory Planning. Robotics and Autonomous Systems. Paises Bajos: NorthHolland Publishing Co., vol. 60, no. 12, pp. 1457-1471. ISSN 0921-8890.

[16] KIM, Hanguen; KIM, Donghoon; SHIN, JaeUk; KIM, Hyongjin; MYUNG, Hyun, Julio 2014. Angular rate-Constrained Path Planning Algorithm for Unmanned Surface Vehicles. Ocean Engineering. Elsevier, vol. 84, no. 1, pp. 37-44. ISSN 0029-8018.

[17] TAM, CheeKuang; BUSCKNALL, Richard, Septiembre 2010. Collision Risk Assessment for Ships. Journal of Marine Science and Technology. Japan: Japan Society of Naval Architects and Ocean Engineers (JASNAOE) $\&$ Springer, vol. 15 , no. 3 , pp. 257-270. ISSN 0948-4280.

[18] YANG, Joe-Ming; TSENG, Chien-Ming; TSENG, P.S., Enero 2015. Path Planning on Satellite Images for Unmanned Surface Vehicles. International Journal of Naval Architecture and Ocean Engineering. Corea: Society of Naval Architects of Korea, vol. 7, no. 1, pp. 87-99. ISSN 2092-6782.

[19] LUMPKIN, R.; JOHNSON, G.; GARZOLI, S.; PAZOS, M.; REDMAN, J.; GARRAFFO, Z., 2014.A drifter-derived seasonal climatology of global near-surface currents. National Oceanic and Atmospheric Administration (U.S. Department of Commerce [en línea]. Disponible en: http://www.aoml.noaa. gov/phod/dac/dac_meanvel.php [Consulta: $24 / 02 / 2016]$.

[20] LUMPKIN, Rick; JOHNSON, Gregory C., Junio 2013. Global ocean surface velocities from drifters : Mean, variance, El Niño - Southern Oscillation response, and seasonal cycle. Journal of Geophysical Research: Oceans. Wiley, vol. 118, no. 6, pp. 2992-3006. ISSN 0148-0227. 